\title{
Characterization of stochastic orders by L-functionals ${ }^{1}$
}

\author{
Miguel A. Sordo ${ }^{1}$, Héctor M. Ramos ${ }^{2}$ \\ ${ }^{1}$ Departamento de Estadística e I. O., Facultad de Ciencias Económicas y Empresar- \\ iales, Duque de Nájera, 8, 11002 Cádiz, Spain. E-mail: mangel.sordo@uca.es \\ 2 Departamento de Estadística e I. O., Facultad de Ciencias Económicas y Empresar- \\ iales, Duque de Nájera, 8, 11002 Cádiz, Spain. E-mail: hector.ramos@uca.es
}

Random variables may be compared with respect to their location by comparing certain functionals ad hoc, such as the mean or median, or by means of stochastic ordering based directly on the properties of the corresponding distribution functions. These alternative approaches are brought together in this paper. We focus on the class of L-functionals discussed by Bickel and Lehmann (1975) and characterize the comparison of random variables in terms of these measures by means of several stochastic orders based on iterated integrals, including the increasing convex order.

\section{Introduction}

A common problem in many fields that use the theory of probability as a tool is to compare two random variables with respect to aspects such as dispersion and location. The simplest way of doing this is by comparing functionals of the random variables, such as dispersion and location measures. This makes cardinal comparisons based on only two single numbers and, therefore, is often not very informative. In addition, when two random variables are compared by means of one or a few measures, such as the mean or standard deviation, choices can be made from a large set of similar functionals and some may produce contradictory conclusions. The arbitrariness of choice is greatly reduced if the verdict of comparisons holds for a class of measures that satisfy a set of reasonable axioms rather than a single measure. This approach leads to ordinal rather than cardinal comparisons. Therefore, if, for example, $X$ and $Y$ are two random variables with respective distribution functions $F$ and $G$, and we consider the class of functionals $I_{\omega}$ given by

$$
I_{\omega}(X)=\int_{-\infty}^{+\infty} \omega(t) d F(t)
$$

\footnotetext{
${ }^{1}$ Preprint, final version published in Statistical Papers 48, 249-263 (2007)
} 
where $\omega$ is a real function, we say that $X$ is smaller than $Y$ in the usual stochastic (respectively, increasing convex) order, if and only if

$$
I_{\omega}(X) \leq I_{\omega}(Y) \text { for all } \omega \in \Omega
$$

where $\Omega$ is the class of increasing (respectively increasing convex) functions for which the functionals exist (throughout this paper "increasing" means "nondecreasing"). Standard references for these orders, denoted by $X \leq_{s t} Y$ (respectively, $X \leq_{i c x} Y$ ) are Ross (1983), Stoyan (1983) and Shaked and Shanthikumar (1994). It is an immediate consequence of the definition that $X \leq_{s t} Y$ implies $X \leq_{i c x} Y$.

Let $F^{-1}$ be the corresponding left continuous inverse of $F$, defined by

$$
F^{-1}(t)=\inf \{x: F(x) \geq t\},
$$

$\left(F^{-1}\right.$ is also called the quantile function of $\left.X\right)$. Characterizations of stochastic orders by integrals in which the quantile functions appear can be found in Arnold (1987), Muliere and Scarsini (1989), Wang and Young (1998), Fagiuoli et al. (1999), Ogryczak and Ruszczynski (2002) and Ramos and Sordo (2002), among others.

Our main concern in this paper is the search for conditions under which two random variables can be unanimously ranked by large classes of functionals of the form

$$
T_{\Phi}(X)=\int_{0}^{1} F^{-1}(t) d \Phi(t)
$$

where $\Phi$ is any distribution function on $(0,1)$. The class $C$ of functionals of the form (2) provides a large and important family of statistical measures that have been discussed in the literature (see, for example, Shorack (1972), Bickel and Lehmann (1975), Sendler (1979), or Serfling (1980), among others). The members of $C$ satisfy the axioms of Bickel and Lehmann (1975) for all measures of location. Important particular cases of (2) are the median and other quantiles (obtained when $\Phi(t)$ is the distribution function of the probability measure concentrated at a point) and the trimmed expectations defined by

$$
\frac{1}{\beta-\alpha} \int_{\alpha}^{\beta} F^{-1}(t) d t,
$$

with $0 \leq \alpha<\beta \leq 1$. The mean is the limiting case corresponding to $\alpha=0$ and $\beta=1$. When $T_{\Phi}(X)$ is evaluated at the empirical distribution of a sample, (2) takes the form of an L-statistic, i.e., a linear combination of order statistics (Serfling (1980), provides many examples). By analogy to L-statistics, functionals of the form (2) are sometimes referred to as L-functionals (Serfling (1980), Bickel and Lehmann (1975), Giovagnoli and Regoli (1993)). L-statistics often represent estimators of the corresponding L-functionals (see, for example, Bickel and Lehmann (1975), Andrews et al. (1972), and Huber (1972)) and asymptotic normality results are available under various restrictions on $F$ and 
$\Phi$ (see Shorack (1972), Sendler (1979), Serfling (1980), and Shorack and Wellner (1986)). Bickel and Lehmann (1975) proved that the measures defined by (2) are robust under mild conditions.

It is easy to see that $X \leq_{s t} Y$ holds, if and only if $F^{-1}(t) \leq G^{-1}(t)$ for all $t \in[0,1]$, or, equivalently, if

$$
\int_{0}^{1} F^{-1}(t) d \Phi_{p}(t) \leq \int_{0}^{1} G^{-1}(t) d \Phi_{p}(t),
$$

where $\Phi_{p}(t)$ denotes the distribution function of the probability measure concentrated at $p$. From this observation it follows that

$$
X \leq_{s t} Y \Longleftrightarrow T_{\Phi}(X) \leq T_{\Phi}(Y) \text { for all } T_{\Phi} \in C
$$

for which the functionals exist.

Characterization (3) provides us with a means of cheking the unanimous ordering of random variables in terms of the functionals of $C$ without needing to agree on the form of $\Phi(p)$. However, as is well recognized, stochastic order performs poorly in empirical ranking of distributions because a large proportion of distributions fail to satisfy this condition. In Section 2, the comparisons of random variables according to the measures of

$$
C_{1}=\left\{T_{\Phi} \in C \text { such that } \Phi \text { is convex }\right\}
$$

are characterized in terms of the increasing convex order. This order can rank distributions which stochastic order fails to rank, and therefore is empirically more useful. By requiring additional assumptions on the form of $\Phi(p)$, the comparisons of the members of $C_{1}$ are characterized in terms of an order that is weaker than $\leq_{i c x}$.

In Section 2 we also present a condition for stochastic equality of two random variables under the increasing convex order (this is stated as Theorem 2.2). We prove, in the spirit of a parallel result obtained by Bhattacharjee and Bhattacharya (2000) regarding the increasing convex order and the functionals of the form (1), that if $X \leq_{i c x} Y$ and $T_{\Phi}(X)=T_{\Phi}(Y)$ for some increasing and strictly convex function $\Phi$, then $X$ and $Y$ have the same distribution. Other results in the literature that give conditions, involving various stochastic orders, which imply stochastic equalities, can be found in Baccelli and Makowski (1989), Bhattacharjee and Sethuraman (1990), Scarsini and Shaked (1990), Bhattacharjee (1991), Jun (1994), Li and Zhu (1994), Cai and Wu (1997), Scarsini (1998), Denuit et al. (2000) and Bhattacharjee and Bhattacharya (2000)

The remainder of the paper is organized as follows. The results of Section 2 are applied in Section 3 to characterize the comparison of non-negative random variables according to a class of generalized L-functionals in terms of the socalled $\leq^{(p)}$ order, introduced in Bhattacharjee (1991) (the exact definition of this order is given in Section 3). As a consequence of Theorem 2.2, we obtain a condition for stochastic equality of (p)-ordered random variables which extends some results of Jun (1994) and Li and Zhu (1994). 
Finally, Section 4 contains, as an application of a result in Section 2, the characterization of the dilation order (the exact definition of this order is given in Section 4) by means of the increasing convex order.

As in Ramos and Sordo (2003), some results in this paper are obtained as a consequence of the theory of submajorization, as applied to decreasing rearrangements of functions (on this concept, see Hardy et al. (1929), Ryff (1963), and Chong, (1974)). If we denote by $M(\Omega, \mu)$ the set of all extended real-valued measurable functions on a measure space $(\Omega, \Lambda, \mu)$, the decreasing rearrangement of $f \in M(\Omega, \mu)$ is defined by

$$
f^{*}(t)=\inf \left\{s \in \mathbf{R}: D_{f}(s) \leq t\right\}, t \in[0, \mu(\Omega)]
$$

where

$$
D_{f}(s)=\mu(\{x: f(x)>s\}),
$$

for each $s \in[-\infty, \infty]$. We need the following result from Chong (1974).

Theorem 1.1 Suppose $(\Omega, \Lambda, \mu)$ and $\left(\Omega^{\prime}, \Lambda^{\prime}, \mu^{\prime}\right)$ are measure spaces such that $\mu(\Omega)=\mu^{\prime}\left(\Omega^{\prime}\right)=a<\infty$ and denote by $m$ the Lebesgue measure on $\mathbf{R}$. If $f \in L^{1}(\Omega, \mu)$ and $g \in L^{1}\left(\Omega^{\prime}, \mu^{\prime}\right)$, then

$$
\begin{gathered}
\int_{0}^{t} f^{*} d m \leq \int_{0}^{t} g^{*} d m \text { for all } t \in[0, a] \\
\quad \text { if and only if } \\
\int_{\Omega} \Psi(f) d \mu \leq \int_{\Omega^{\prime}} \Psi(g) d \mu^{\prime}
\end{gathered}
$$

for all increasing convex functions $\Psi: \mathbf{R} \longrightarrow \mathbf{R}$.

\section{Characterizations in terms of the increasing convex order}

An useful characterization of the increasing convex order is given in the following lemma. This result is well known for non-negative random variables, and it already appears for instance in Muliere and Scarsini (1989); however, we provide a proof of it which applies also to random variables that need not be nonnegative.

Lemma 2.1 Let $X$ and $Y$ be two random variables with distribution functions $F$ and $G$, respectively. Then, $X \leq_{i c x} Y$ if and only if

$$
\int_{p}^{1} F^{-1}(t) d t \leq \int_{p}^{1} G^{-1}(t) d t \text { for all } p \in[0,1] .
$$


Proof Let $\bar{F}=1-F$ be. Since $\bar{F}^{-1}(t)=F^{-1}(1-t)$ for all $t \in[0,1]$, a change of variable shows that $(6)$ is equivalent to

$$
\int_{0}^{p} \bar{F}^{-1}(t) d t \leq \int_{0}^{p} \bar{G}^{-1}(t) d t \text { for all } p \in[0,1] .
$$

Now, let $\left(\Omega_{X}, \mathcal{B}_{X}, P_{X}\right)$ and $\left(\Omega_{Y}, \mathcal{B}_{Y}, P_{Y}\right)$ be the probability spaces on which $X$ and $Y$, respectively, are defined. Define $f(\omega)=X(\omega)$ for all $\omega \in \Omega$ and $g(\omega)=Y(\omega)$ for all $\omega \in \Omega_{Y}$. Then,

$$
D_{f}(x)=P_{X}\left\{\omega \in \Omega_{X}: f(\omega)>x\right\}=\bar{F}(x), \text { for all } x \in \mathbf{R},
$$

and, analogously,

$$
D_{g}(x)=\bar{G}(x) \text {, for all } x \in \mathbf{R} .
$$

The decreasing rearrangements of $f$ and $g$ are given, respectively, by $f^{*}(t)=$ $\bar{F}^{-1}(t)$ and $g^{*}(t)=\bar{G}^{-1}(t)$, for all $t \in[0,1]$. From Theorem 1.1 it follows that (7) holds if and only if

$$
\int_{\Omega_{X}} \Psi(X) d P_{X} \leq \int_{\Omega_{Y}} \Psi(Y) d P_{Y}
$$

for all increasing and convex functions $\Psi: \mathbf{R} \longrightarrow \mathbf{R}$, which is $X \leq_{i c x} Y$.

The main results of this section follow. Recall that $C_{1}$ is the class of functionals $T_{\Phi} \in C$ such that $\Phi$ is convex. The following result characterizes the comparison of random variables according to functionals of $C_{1}$ in terms of increasing convex order.

Theorem 2.1 Let $X$ and $Y$ be two random variables with finite means. Then,

$$
X \leq_{i c x} Y \text { if and only if } T_{\Phi}(X) \leq T_{\Phi}(Y) \text { for all } T_{\Phi} \in C_{1} .
$$

Proof $(\Longrightarrow)$ Let $F$ be the distribution function of $X$ and let $\Phi:[0,1] \longrightarrow \mathbf{R}$ be an increasing and convex function. Then, there exists an increasing, nonnegative and integrable function $\phi$ such that

$$
\Phi(p)-\Phi(0)=\int_{0}^{p} \phi(t) d t, \quad p \in[0,1)
$$

(Zygmund, 1959). Consequently, using the properties of the Riemann-Stieltjes integral (see Apostol, (1973)), we have

$$
T_{\Phi}(X)=\int_{0}^{1} F^{-1}(p) d \Phi(p)=-\int_{0}^{1} \phi(p) d F_{2}^{-1}(p)
$$

where

$$
F_{2}^{-1}(p)=\int_{p}^{1} F^{-1}(t) d t, p \in[0,1]
$$


Partial integration of (8) and $F_{2}^{-1}(1)=0$ produces the following alternative expression for (2):

$$
T_{\Phi}(X)=\phi(0) F_{2}^{-1}(0)+\int_{0}^{1} F_{2}^{-1}(p) d \phi(p) .
$$

Analogously, if $G$ denotes the distribution function of $Y$, we have

$$
T_{\Phi}(Y)=\phi(0) G_{2}^{-1}(0)+\int_{0}^{1} G_{2}^{-1}(p) d \phi(p),
$$

where

$$
G_{2}^{-1}(p)=\int_{p}^{1} G^{-1}(t) d t, p \in[0,1] .
$$

Since $X \leq_{i c x} Y$, it follows from Lemma 2.1 that

$$
F_{2}^{-1}(p) \leq G_{2}^{-1}(p) \text { for all } p \in[0,1]
$$

and, therefore, the inequality

$$
\phi(0) F_{2}^{-1}(0)+\int_{0}^{1} F_{2}^{-1}(p) d \phi(p) \leq \phi(0) G_{2}^{-1}(0)+\int_{0}^{1} G_{2}^{-1}(p) d \phi(p)
$$

is a consequence of (13), $\phi(0) \geq 0$ and $d \phi(p) \geq 0$ (the increasing nature of $\phi$ ensures that the increments $d \phi$ are non-negative). Combining (10), (11) and (14) it is seen that $T_{\Phi}(X) \leq T_{\Phi}(Y)$ is satisfied.

$(\Longleftarrow)$ Because the function $\Phi_{p}(t)$ defined by

$$
\Phi_{p}(t)=\left\{\begin{array}{ccc}
0 & \text { si } & t<p \\
t-p & \text { si } & t \geq p
\end{array}\right.
$$

is an increasing and convex function of $t$ for each $p \in[0,1]$, we have

$$
\int_{0}^{1} F^{-1}(t) d \Phi_{p}(t) \leq \int_{0}^{1} G^{-1}(t) d \Phi_{p}(t) \text { for all } p \in[0,1]
$$

or, equivalently,

$$
\int_{p}^{1} F^{-1}(t) d t \leq \int_{p}^{1} G^{-1}(t) d t \text { for all } p \in[0,1],
$$

which is $X \leq_{i c x} Y$ by Lemma 2.1 .

The next result gives a characterization for stochastic equality of two random variables under the increasing convex order.

Theorem 2.2 Let $X$ and $Y$ be two random variables with finite means. If $X \leq_{i c x} Y$ and if

$$
T_{\Phi}(X)=T_{\Phi}(Y)
$$


for some increasing and strictly convex function $\Phi$, then $X$ and $Y$ have the same distribution.

Proof Suppose that $\Phi$ is increasing and strictly convex and that $X \leq_{i c x} Y$. Since $\Phi$ is strictly convex, its derivative $\phi$ is strictly increasing. Combining (10), (11) and (16) it follows that

$$
\phi(0)\left[G_{2}^{-1}(0)-F_{2}^{-1}(0)\right]+\int_{0}^{1}\left[G_{2}^{-1}(p)-F_{2}^{-1}(p)\right] d \phi(p)=0 .
$$

From (17) it follows that both $\phi(0)\left[G_{2}^{-1}(0)-F_{2}^{-1}(0)\right]=0$ and

$$
\int_{0}^{1}\left[G_{2}^{-1}(p)-F_{2}^{-1}(p)\right] d \phi(p)=0
$$

hold, since the left hand sides of both expressions are non-negative. From (13) and (18) it follows that $G_{2}^{-1}(p)=F_{2}^{-1}(p)$ almost everywhere on $[0,1]$. Now, we claim that $G_{2}^{-1}(p)=F_{2}^{-1}(p)$ for all $p \in[0,1]$. Suppose, by contradiction, that $G_{2}^{-1}\left(p_{0}\right)>F_{2}^{-1}\left(p_{0}\right)$ for some $p_{0} \in[0,1]$. Then, there exists an interval $(a, b) \subset[0,1]$ such that $p_{0} \in(a, b)$ and $G_{2}^{-1}(p)>F_{2}^{-1}(p)$ for all $p \in(a, b)$, since both $F_{2}^{-1}$ and $G_{2}^{-1}$ are, by definition, continuous on $[0,1]$. However

$$
\int_{0}^{1}\left[G_{2}^{-1}(p)-F_{2}^{-1}(p)\right] d \phi(p) \geq \int_{a}^{b}\left[G_{2}^{-1}(p)-F_{2}^{-1}(p)\right] d \phi(p)>0
$$

since $\phi$ is strictly increasing, and this contradicts (18). Therefore

$$
\int_{p}^{1} F^{-1}(t) d t=\int_{p}^{1} G^{-1}(t) d t, \text { for all } p \in[0,1] .
$$

Differentiating (19) we obtain $F^{-1}=G^{-1}$, that is, $X$ and $Y$ have the same distribution.

From Theorem 2.2 we obtain the following corollary.

Corollary 2.1 Let $X_{1}, X_{2, \ldots, X_{n}}(n \geq 2)$ be a collection of independent and identically distributed random variables, and let $Y_{1}, Y_{2}, \ldots, Y_{n}(n \geq 2)$ be another collection of independent and identically distributed random variables. If $X_{1} \leq_{i c x} Y_{1}$ and

$$
E\left[\max \left\{X_{1}, X_{2, \ldots}, X_{n}\right\}\right]=E\left[\max \left\{Y_{1}, Y_{2}, \ldots, Y_{n}\right\}\right]
$$

then $X_{1}$ and $Y_{1}$ have the same distribution.

Proof Let $F$ and $G$ denote the distribution functions of $X_{1}$ and $Y_{1}$, respectively. By taking $\Phi(t)=t^{n}$, we obtain

$$
E\left[\max \left\{X_{1}, X_{2, \ldots,} X_{n}\right\}\right]=\int_{0}^{1} F^{-1}(t) d \Phi(t)
$$


and

$$
E\left[\max \left\{Y_{1}, Y_{2}, \ldots, Y_{n}\right\}\right]=\int_{0}^{1} G^{-1}(t) d \Phi(t) .
$$

The result now follows from Theorem 2.2.

Let $F_{2}^{-1}(t)$ and $G_{2}^{-1}(t)$ be the curves given by (9) and (12), respectively. We have shown in Theorem 2.1 that non-intersection of these curves is equivalent to the unanimous ordering generated by the class of functionals of $C_{1}$. In addition, since each $\Phi \in C_{1}$ is convex, its derivative $\Phi^{\prime}$ exists (except possibly at a countable number of points) and we can obtain a result that allows one to rank two random variables whose associated curves intersect according to the functionals of $C_{1}$, by restricting such derivative to be convex. Use the term $C_{1.1}$ for the class of functionals of the form (2) where $\Phi$ is increasing, convex and differentiable almost everywhere (a.e.) with convex derivative, that is,

$$
C_{1.1}=\left\{T_{\Phi} \in C_{1} \text { such that } \phi \text { is convex, where } \Phi^{\prime}(x)=\phi \text { a.e. }\right\} \text {. }
$$

Theorem 2.3 Let $X$ and $Y$ be two random variables with means $\mu_{X}$ and $\mu_{Y}$, respectively. Then,

$$
\int_{x}^{1} F_{2}^{-1}(p) d p \leq \int_{x}^{1} G_{2}^{-1}(p) d p \text { for all } x \in[0,1] \text { and } \mu_{X} \leq \mu_{Y}
$$

if and only if

$$
T_{\Phi}(X) \leq T_{\Phi}(Y) \text { for all } T_{\Phi} \in C_{1.1} .
$$

Proof First we prove the sufficiency condition. It is obvious that (21) implies $\mu_{X} \leq \mu_{Y}$. Now, for each $p \in[0,1]$, the function

$$
\Phi_{p}(x)=\int_{0}^{x}(t-p)^{+} d t
$$

where $(x)^{+}=\max \{x, 0\}$, is increasing, convex and has a convex derivative. Therefore,

$$
\begin{gathered}
T_{\Phi_{p}}(X)=\int_{0}^{1} F^{-1}(t) d \Phi_{p}(t)= \\
=-\int_{0}^{1}(t-p)^{+} d F_{2}^{-1}(t)=-\left[\int_{p}^{1}(t-p) d F_{2}^{-1}(t)\right]
\end{gathered}
$$

belong to $C_{1.1}$. Since $F_{2}^{-1}(1)=0$, integration by parts yields

$$
T_{\Phi_{p}}(X)=\int_{p}^{1} F_{2}^{-1}(t) d t
$$

and the result follows. 
Now we prove the necessary condition. Let $T_{\Phi} \in C_{1.1}$. We can, therefore, assume that $\Phi^{\prime}=\phi$ a.e. for some increasing convex and non-negative function $\phi$. Then, there exists an increasing and non-negative function $\lambda$ such that

$$
\phi(p)-\phi(0)=\int_{0}^{p} \lambda(x) d x=\int_{0}^{1}(p-x)^{+} d \lambda(x)+p \lambda(0), p \in[0,1),
$$

where the second equality follows from integration by parts. Using the properties of the Riemann-Stieltjes again, we have

$$
T_{\Phi}(X)=\int_{0}^{1} F^{-1}(t) d \Phi(t)=-\int_{0}^{1} \phi(p) d F_{2}^{-1}(p) .
$$

Combining (22) with (23) we obtain

$$
T_{\Phi}(X)=-\int_{0}^{1}\left[\phi(0)+\int_{0}^{1}(p-x)^{+} d \lambda(x)+p \lambda(0)\right] d F_{2}^{-1}(p) .
$$

Using the additivity properties of integrals, Fubini's theorem and that $F_{2}^{-1}(0)=$ $\mu_{X}$ and $F_{2}^{-1}(1)=0$, it follows that (24) can be rewritten as

$$
\begin{gathered}
T_{\Phi}(X)=\phi(0) \mu_{X}-\int_{0}^{1}\left[\int_{0}^{1}(p-x)^{+} d F_{2}^{-1}(p)\right] d \lambda(x) \\
-\lambda(0) \int_{0}^{1} p d F_{2}^{-1}(p) .
\end{gathered}
$$

Integration by parts yields

$$
\int_{0}^{1}(p-x)^{+} d F_{2}^{-1}(p)=-\int_{x}^{1} F_{2}^{-1}(p) d p
$$

and

$$
\int_{0}^{1} p d F_{2}^{-1}(p)=-\int_{0}^{1} F_{2}^{-1}(p) d p
$$

and, combining this with (25), we have

$$
T_{\Phi}(X)=\phi(0) \mu_{X}+\int_{0}^{1}\left[\int_{x}^{1} F_{2}^{-1}(p) d p\right] d \lambda(x)+\lambda(0) \int_{0}^{1} F_{2}^{-1}(p) d p .
$$

Finally, taking into account that $\phi(0) \geq 0, \lambda(0) \geq 0$ and $d \lambda(x) \geq 0$, from (20) the result follows.

Remark 2.1 Functionals of $C$ appear in the theory of choice under uncertainty (see, for example, Yaari, 1987, and Röel, 1987). In this framework, a random variable $X$ represents a random loss and (2) is used to evaluate the risk associated to $X$. In this context, for non-negative random variables, Wang and Young (1998) obtained two results that correspond to our theorems 2.1 and 2.3. However, it should be mentioned that in these results, the weight function $\Phi$ 
in (2) is assumed to be differentiable, and thus inadequate for expressing some location measures mentioned in Section 1. On the other hand, note that all the results in this section can be reworded in terms of concave rather than convex functions. This can be easily seen by taking into account that

$$
T_{\Phi}(X)=-T_{\widetilde{\Phi}}(-X)
$$

with $\widetilde{\Phi}(t)=-\Phi(1-t)$ (note that $\Phi$ is increasing and convex if and only if $\widetilde{\Phi}$ is increasing and concave).

\section{Characterizations in terms of the order $\leq^{(p)}$}

Let $X$ and $Y$ be two nonnegative random variables with survival functions $\bar{F}$ and $\bar{G}$, respectively. For $p>0$, we say that $X \leq^{(p)} Y$ if

$$
\int_{t}^{+\infty} x^{p-1} \bar{F}(x) d x \leq \int_{t}^{+\infty} x^{p-1} \bar{G}(x) d x, \text { for all } t \geq 0
$$

provided the integrals exist.

The partial ordering $\leq^{(p)}$ was introduced and studied by Bhattacharjee (1991). Li and Zhu (1994), Jun (1994) and Cai and Wu (1997) provided some applications in the context of reliability theory. For nonnegative random variables, is easy to see that

$$
X \leq^{(p)} Y \Longleftrightarrow X^{p} \leq_{i c x} Y^{p}
$$

The following result characterizes the comparison of random variables according to the members of a class of generalized L-functionals in terms of the $\leq{ }^{(p)}$ order.

Corollary 3.1 Let $X$ and $Y$ be two nonnegative random variables with finite means and distribution functions $F$ and $G$, respectively. Then, $X \leq{ }^{(p)} Y$ if and only if

$$
\int_{0}^{1}\left[F^{-1}(t)\right]^{p} d \Phi(t) \leq \int_{0}^{1}\left[G^{-1}(t)\right]^{p} d \Phi(t)
$$

for all distribution function $\Phi$ convex on $(0,1)$.

Proof Let $F_{p}^{-1}(t)$ be the quantile function associated to the random variable $X^{p}$. Using (27) and taking into account that

$$
\int_{0}^{1} F_{p}^{-1}(t) d \Phi(t)=\int_{0}^{1}\left[F^{-1}(t)\right]^{p} d \Phi(t)
$$

the result follows easily from Theorem 2.1 .

The following result gives a condition for stochastic equality under the ordering $\leq^{(p)}$. Other characterizations of stochastic equality under this order can be found in Jun (1994), Li and Zhu (1994) and Cai and Wu (1997). 
Corollary 3.2 Let $X$ and $Y$ be two nonnegative random variables with finite means and distribution functions $F$ and $G$, respectively. If $X \leq^{(p)} Y$ and

$$
\int_{0}^{1}\left[F^{-1}(t)\right]^{r} d \Phi(t)=\int_{0}^{1}\left[G^{-1}(t)\right]^{r} d \Phi(t)
$$

for some $r \geq p$ and some increasing and strictly convex function $\Phi$, then $X$ and $Y$ have the same distribution.

Proof It is well known (Ross, 1983) that if $X \leq_{i c x} Y$ then $g(X) \leq_{i c x} g(Y)$ for all increasing convex function $g$ on $\mathbf{R}$. Since $g(x)=x^{r}, r \geq 1, x>0$, is an increasing and convex function of $x$, it follows from (27) that $X \leq^{(p)} Y$ implies

$$
X^{r} \leq_{i c x} Y^{r} \text { for all } r \geq p .
$$

Now, combining (29) and (28), the result follows from Theorem 2.2.

Application 3.1 Following Bickel and Lehmann (1975), a large and important class of dispersion measures for symmetric distributions is provided, for each $p \geq 1$, by the class of functionals

$$
J_{\Phi}(X)=\left\{\int_{0}^{1}\left[F_{\left|X-\mu_{X}\right|}^{-1}(t)\right]^{p} d \Phi(t)\right\}^{\frac{1}{p}}
$$

where $F$ is assumed to be symmetric about $\mu_{X}, F_{\left|X-\mu_{X}\right|}$ denotes the distribution function of $\left|X-\mu_{X}\right|$ and $\Phi$ is any probability distribution on $(0,1)$. Particular members of this class are the standard deviation of $X$, given by (30) with $p=2$ and $\Phi$ the uniform distribution on $(0,1)$, the doubly trimmed standard deviation, given by (30) with $p=2$ and $\Phi$ the uniform distribution on $(\alpha, 1-\beta)$ and the $\alpha$ th quantile, obtained from (30) by letting $\Phi$ assign probability 1 to the point $\alpha$ (this measure is independent of $p$ ). A generalization of the standard deviation is the $\gamma$ th power deviation obtained by replacing $p$ by $\gamma$ in (30) and letting $\Phi$ be the uniform distribution on $(0,1)$.

It follows from Corollary 3.1 that if $X$ is a random variable whose distribution function $F$ is symmetric about $\mu_{X}$, and $Y$ is another random variable whose distribution function $G$ is symmetric about $\mu_{Y}$, then, for each $p \geq 1$, we have

$$
\left|X-\mu_{X}\right| \leq^{(p)}\left|Y-\mu_{Y}\right|
$$

if and only if

$$
J_{\Phi}(X) \leq J_{\Phi}(Y) \text { for all } \Phi \text { convex on }(0,1) .
$$

From this characterization we think that the $\leq^{(p)}$ order will play an important role in the comparisons of symmetric random variables in terms of dispersion.

Application 3.2 Let $X_{1}, X_{2}, \ldots, X_{n}(n \geq 2)$ be a collection of independent, non-negative and identically distributed random variables, and let $Y_{1}, Y_{2}, \ldots, Y_{n}$ $(n \geq 2)$ another collection of independent, non-negative and identically distributed random variables $\left(X_{i}\right.$ and $Y_{i}$ can be thought of as the lifetimes of the $i$ th 
component of two systems respectively, for $i=1,2, \ldots, n)$. Let $F$ and $G$ denote the distribution functions of $X_{1}$ and $Y_{1}$, respectively. By taking $\Phi(t)=t^{n}$, we obtain

$$
E\left[\max \left\{X_{1}^{r}, X_{2, \ldots,}^{r}, X_{n}^{r}\right\}\right]=\int_{0}^{1}\left[F^{-1}(t)\right]^{r} d \Phi(t)
$$

and

$$
E\left[\max \left\{Y_{1}^{r}, Y_{2, \ldots,}^{r}, Y_{n}^{r}\right\}\right]=\int_{0}^{1}\left[G^{-1}(t)\right]^{r} d \Phi(t) .
$$

It follows from Corollary 3.2 that if $X \leq^{(p)} Y$ and

$$
E\left[\max \left\{X_{1}^{r}, X_{2, \ldots}^{r}, X_{n}^{r}\right\}\right]=E\left[\max \left\{Y_{1}^{r}, Y_{2, \ldots}^{r}, Y_{n}^{r}\right\}\right]
$$

for some $r \geq p$, then $X_{1}$ and $Y_{1}$ have the same distribution. A different proof of this result when $X_{1}$ (or $Y_{1}$ ) has a support of the form $(0, \infty)$ can be found in Jun (1994).

\section{Application to the characterization of the dilation order in terms of the increasing convex order}

Let $X$ and $Y$ be two random variables with respective finite means $\mu_{X}$ and $\mu_{Y}$. If $X-\mu_{X} \leq_{i c x} Y-\mu_{Y}$, then $X$ and $Y$ are said to be ordered according to the dilation order (denoted by $X \leq_{d i l} Y$ ). It follows from Lemma 2.1 that $X \leq_{d i l} Y$ if and only if

$$
\int_{p}^{1}\left[F^{-1}(x)-\mu_{X}\right] d x \leq \int_{p}^{1}\left[G^{-1}(x)-\mu_{Y}\right] d x, \text { for all } p \in(0,1),
$$

(this is also proven in Ramos and Sordo (2003)). Fagiuoli et al. (1999) proved that $X \leq_{d i l} Y$ if and only if

$$
E\left[X-\mu_{X} \mid X \geq F^{-1}(p)\right] \leq E\left[Y-\mu_{Y} \mid Y \geq G^{-1}(p)\right] \text { for all } p \in[0,1) .
$$

In addition, $X \leq_{d i l} Y$ if and only if

$$
E\left[X-\mu_{X} \mid X \leq F^{-1}(p)\right] \geq E\left[Y-\mu_{Y} \mid Y \geq G^{-1}(p)\right] \text { for all } p \in(0,1] .
$$

The following result is a natural extension of these characterizations.

Theorem 4.1 Let $X$ and $Y$ be two random variables with finite respective means $\mu_{X}$ and $\mu_{Y}$. Then, $X \leq_{d i l} Y$ if and only if

$$
\left\{X-\mu_{X} \mid X \geq F^{-1}(p)\right\} \leq_{i c x}\left\{Y-\mu_{Y} \mid Y \geq G^{-1}(p)\right\} \text { for all } p \in[0,1),
$$

if and only if

$$
\left\{X-\mu_{X} \mid X \leq F^{-1}(p)\right\} \geq_{i c x}\left\{Y-\mu_{Y} \mid Y \leq G^{-1}(p)\right\} \text { for all } p \in(0,1] .
$$

Proof We prove that $X \leq_{d i l} Y$ if and only if (33) holds (the equivalence with 
(34) is similarly proven). For each $p \in[0,1)$, denote the distribution function of

$$
\left\{X-\mu_{X} \mid X \geq F^{-1}(p)\right\}
$$

by $F_{p}$ and let $F_{p}^{-1}$ be the corresponding left inverse distribution function. Then,

$$
F_{p}(x)=\left\{\begin{array}{cc}
0 & x<F^{-1}(p) \\
\frac{F\left(x+\mu_{X}\right)-p}{1-p} & x \geq F^{-1}(p)
\end{array}\right.
$$

and

$$
F_{p}^{-1}(t)=F^{-1}[p+(1-p) t]-\mu_{X}, \text { for all } t \in(0,1) .
$$

Similarly, we obtain

$$
G_{p}^{-1}(t)=G^{-1}[p+(1-p) t]-\mu_{Y}, \text { for all } t \in(0,1) .
$$

From (35), (36) and Lemma 2.1 it follows that (33) holds if and only if

$$
\begin{gathered}
\int_{u}^{1}\left(F^{-1}[p+(1-p) t]-\mu_{X}\right) d t \leq \\
\leq \int_{u}^{1}\left(G^{-1}[p+(1-p) t]-\mu_{Y}\right) \text {, for all } u \in(0,1) \text {, for all } p \in(0,1) .
\end{gathered}
$$

A change of variable shows that (37) holds if and only if (31) holds, and this means $X \leq_{\text {dil }} Y$.

To see the usefulness of Theorem 4.1, note that the increasing convex order is much more informative than a simple inequality between expected values, as in (32).

When $\mu_{X}=\mu_{Y}$, the dilation order reduces to the convex order (denoted $\leq_{c x}$; see Shaked and Shanthikumar (1994)). As an immediate consequence of Theorem 4.1 we have the following result.

Corollary 4.1 Let $X$ and $Y$ be two random variables with equal finite means. Then, $X \leq_{c x} Y$ if and only if

$$
\left\{X \mid X \geq F^{-1}(p)\right\} \leq_{i c x}\left\{Y \mid Y \geq G^{-1}(p)\right\} \text { for all } p \in[0,1),
$$

and if and only if

$$
\left\{X \mid X \leq F^{-1}(p)\right\} \geq_{i c x}\left\{Y \mid Y \leq G^{-1}(p)\right\} \text { for all } p \in(0,1] .
$$

Acknowledgement We are grateful to the referees for their suggestions

\section{References}

1. Andrews DF, Bickel PJ, Hampel FR, Hubert PJ, Rogers WH, Tukey JW (1972) Robust estimation of location. Princeton Univ. Press, Princeton NJ 
2. Apostol TH (1973) Mathematical Analysis (6th pr.). Reading: Addison-Wesley

3. Arnold BC (1987) Majorization and the Lorenz order: a brief introduction. Springer-Verlag, New York, NY

4. Baccelli F, Makowski AM (1989) Multi-dimensional stochastic ordering and associated random variables. Oper. Res. 37, 478-487

5. Bhattacharjee MC, Sethuraman J (1990) Families of life distributions characterizated by two moments. J. Appl. Probab. 27, 720-725

6. Bhattacharjee MC (1991) Some generalized variability orderings among life distributions with reliability applications. J. Appl. Prob. 28, 374-383

7. Bhattacharjee MC, Bhattacharya RN (2000) Stochastic equivalence of convex ordered distributions and applications. Probab. Engrg. Inform. Sci. 14, 33-48

8. Bickel PJ, Lehmann EL (1975) Descriptive statistics for nonparametric models. I. Introduction. Ann. Statist. 3, 1038-1044. II. Location. Ann. Statist. 3, 1045-1069

9. Bickel PJ, Lehmann EL (1976) Descriptive statistics for nonparametric models. III. Dispersion. Ann. Statist. 4, 1139-1158

10. Cai J, Wu Y (1997) Characterization of life distributions under some generalized stochastic orderings. J. Appl. Probab. 34, 711-719

11. Chong KM (1974) Some extensions of a theorem of Hardy, Littlewood and Pólya and their applications. Canad. J. Math. 26, 1321-1340

12. Denuit M, Lefèvre C, Shaked M (2000) On the theory of high convexity stochastic orders. Statist. Probab. Lett. 47, 287-293

13. Fagiuoli E, Pellerey F, Shaked M (1999) A characterization of the dilation order and its applications. Statist. Papers 40, 393-406

14. Giovagnoli A, Regoli G (1993) Some results on the representation of measures of location and spread as L-functionals. Statist. Probab. Lett. 16, 269-278

15. Hardy GH, Littlewood JE, Pólya G (1929) Some simple inequalities satisfied by convex functions. Mess. Math. 58, 145-152

16. Hickey RJ (1986) Concepts of dispersion in distributions: a comparative note. J. Appl. Probab. 23, 914-921

17. Huber, PJ (1972) Robust statistics: a review. Ann. Math. Statist. 43, 1041-1067

18. Jun C (1994) Characterizations of life distributions by moments of extremes and sample mean. J. Appl. Prob. 31, 148-155 
19. Li H, Zhu H (1994) Stochastic equivalence of ordered random variables with applications in reliability theory. Statist. Probab. Lett. 20, 383-393

20. Muliere P, Scarsini M (1989) A note on stochastic dominance and inequality measures. J. Econ. Theory 49, 314-323

21. Ogryczak W, Ruszczynski A (2002) Dual stochastic dominance and related mean-risk models. SIAM J. Optim. 13, 60-78

22. Ramos HM, Sordo MA (2002) Characterizations of inequality orderings by means of dispersive orderings. Qüestiió 26, 15-28

23. Ramos HM, Sordo MA (2003) Dispersion measures and dispersive orderings. Statist. Probab. Lett. 61, 123-131

24. Röel, A (1987) Risk aversion in Quiggin and Yaari's rank-order model of choice under uncertainty. Economic J. 97, 143-159.

25. Ross SM (1983) Stochastic Processes, Wiley, New York.

26. Ryff JV (1963) On the representation of doubly stochastic operators. Pacific J. Math. 13, 1379-1386

27. Scarsini M, Shaked M (1990) Some conditions for stochastic equality. Naval Res. Logist. 37, 617-625

28. Scarsini M (1998) Multivariate convex orderings, dependence, and stochastic equality. J. Appl. Probab. 35, 93-103

29. Sendler W (1979) On statistical inference in concentration measurement. Metrika 26, 109-122

30. Serfling RJ (1980) Approximation Theorems of Mathematical Statistics. Wiley, New York

31. Shaked M, Shanthikumar JG (1994) Stochastic Orders and their Applications. Academic Press, San Diego, CA

32. Shorack GR (1972) Functions of Order Statistics. Ann. Math. Statist. 43, $412-427$

33. Shorack GR, Wellner JA (1986) Empirical Processes with Applications to Statistics. Wiley, New York

34. Stoyan D (1983) Comparison Methods for Queues and Other Stochastic Models. John Wiley, New York

35. Wang S, Young VR (1998) Ordering risks: Expected utility theory versus Yaari's dual theory of risk. Insurance: Math. and Econ. 22,145-161

36. Yaari, ME (1987) The dual theory of choice under risk. Econometrica 55, 95-115

37. Zygmund A (1959) Trigonometric Series, Vol. I, Cambridge 